\title{
Optimized intrapleural cisplatin chemotherapy with a fibrin carrier after extrapleural pneumonectomy: A preclinical study
}

\author{
Isabelle Opitz, MD, ${ }^{\mathrm{a}}$ Barbara V. Erne, MD, ${ }^{\mathrm{a}}$ Seval Demirbas, ${ }^{\mathrm{a}}$ Alexander Jetter, MD, ${ }^{\mathrm{b}}$ \\ Burkhardt Seifert, MD, ${ }^{\mathrm{c}}$ Rolf Stahel, MD, ${ }^{\mathrm{d}}$ and Walter Weder, MD ${ }^{\mathrm{a}}$
}

\begin{abstract}
Objective: Our objective was to evaluate whether platinum concentrations in chest wall tissue and in serum are optimized by intracavitary application of cisplatin loaded to a fibrin carrier compared with cisplatin solution in a randomized setting of a pig model.
\end{abstract}

\begin{abstract}
Methods: After left-sided pneumonectomy including parietal pleurectomy, pigs were randomly assigned to receive either $90 \mathrm{mg} / \mathrm{m}^{2}$ cisplatin intracavitary solution $(\mathrm{n}=6)$ or to receive $5 \mathrm{mg}$ cisplatin-fibrin $(\mathrm{n}=5)$ applied on a predefined area of the chest wall. Platinum concentration in serum as well as in chest wall tissue was determined at several early time points until day 5 after treatment. Platinum levels were measured by inductively coupled plasma sector field mass spectrometric detection with a matrix-matched calibration procedure.
\end{abstract}

\begin{abstract}
Results: The dose- and surface-corrected (geometric) mean concentration of cisplatin in chest wall tissue 2 hours but also at day 5 after the application was doubled in animals treated with cisplatin-fibrin compared with the animals treated with cisplatin-solution. In serum, the dose- and surface-corrected exposure toward cisplatin (area under the curve ${ }_{0-5 \mathrm{~d}}$ ) was significantly lower with cisplatin-fibrin than with cisplatin-solution $(P<.0005)$. This is also reflected by significantly reduced serum creatinine and urea values in the cisplatinfibrin group $(P<.0001)$. Animals treated with cisplatin-fibrin additionally had a significantly better postoperative course as assessed by a well-being score $(P<.001)$.

Conclusions: After cisplatin-fibrin treatment, cisplatin tissue concentration was increased whereas systemic cisplatin concentrations were significantly reduced in comparison with cisplatin-solution treatment. This finding offers a clear advantage inasmuch as rate and severity of systemic adverse events can be reduced while local cytotoxic concentrations are at least maintained. (J Thorac Cardiovasc Surg 2011;141:65-71)
\end{abstract}

Although overall survival data for patients with malignant pleural mesothelioma were positively influenced by multimodal treatment concepts over the past decade, ${ }^{1,2}$ malignant pleural mesothelioma remains a malignancy with a high rate of local recurrence in a median time around 16 months only. Therefore, new therapeutic strategies to improve local tumor control are required. One particular approach is intracavitary chemotherapy. This application form provides the advantage of easy

From the Divisions of Thoracic Surgery ${ }^{\mathrm{a}}$ and Clinical Pharmacology and Toxicology, ${ }^{\mathrm{b}}$ University Hospital Zurich; the Biostatistics Unit, ${ }^{\mathrm{c}}$ Institute of Social and Preventive Medicine, University of Zurich; and the Laboratories of Molecular Oncology, ${ }^{\mathrm{d}}$ Clinic and Policlinic of Oncology, University Hospital Zurich, Switzerland.

The study was supported by Zürcher Krebsliga.

Disclosures: Authors have nothing to disclose with regard to commercial support.

Read at the 90th Annual Meeting of The American Association for Thoracic Surgery, Toronto, Ontario, Canada, May 1-5, 2010.

I.O. and B.V.E. have equally contributed to this study.

Received for publication May 3, 2010; revisions received Sept 9, 2010; accepted for publication Sept 22, 2010

Address for reprints: Walter Weder, MD, Clinical Director, Division of Thoracic Surgery, University Hospital of Zurich, Rämistrasse 100, 8091 Zurich, Switzerland (E-mail: walter.weder@usz.ch).

0022-5223/\$36.00

Copyright (C) 2011 Published by Elsevier Inc. on behalf of The American Association for Thoracic Surgery

doi:10.1016/j.jtcvs.2010.09.032 accessibility to the large surface of the pleura and the possibility to reduce systemic side effects by increasing the desired local effect. The feasibility of intrapleural cisplatin-based chemotherapy (CDDP) was proven by several groups in clinical phase I or II studies. ${ }^{3-10}$ The results indicate the distinct pharmacokinetic advantage of this treatment with high intrapleural chemotherapeutic drug levels. ${ }^{11}$ However, treatment-related morbidity of up to $49 \%$ and mortality of up to $11 \%$ are reported ${ }^{4,10}$ whereas median overall survivals (11-16 months) do not exceed the those reported for induction chemotherapy plus extrapleural pneumonectomy ( 23 months). ${ }^{1,2}$ One of the major drawbacks of this application form is the rapid absorption of the drug applied, leading to high systemic levels with corresponding side effects such as renal dysfunction. Therefore, further refinement of the intrapleural chemotherapy regimen is required to increase the efficacy of local cisplatin application with maintained dosages and decrease the treatment-related toxicity.

In previous small animal studies, $\mathrm{we}^{12}$ demonstrated higher local concentrations of intracavitary cisplatin (CDDP) if combined with fibrin, whereas systemic concentrations are reduced in comparison to CDDP-solution. Furthermore, we have proven important reduction of tumor recurrence by intracavitary application of cisplatin in an orthotopic 


$$
\begin{aligned}
& \text { Abbreviations and Acronyms } \\
& \text { ANOVA }=\text { analysis of variance } \\
& \text { CDDP }=\text { cisplatin } \\
& \text { CI } \quad=\text { confidence interval } \\
& \text { CV } \quad=\text { coefficient of variation }
\end{aligned}
$$

mesothelioma recurrence model. Tumor recurrence was significantly decreased if CDDP-fibrin or CDDP-solution was applied to the chest cavity after pneumonectomy. ${ }^{13}$

The aim of the present study was to compare platinum local tissue and systemic serum concentrations after pneumonectomy followed by intracavitary application of CDDP-fibrin in comparison with CDDP-solution in a preclinical pig model.

\section{METHODS}

Animal care was provided in accordance with the "Guide for the Care and Use of Laboratory Animals" (National Institutes of Health Publication No. 85-23, revised 1996; Bethesda, Md) and in compliance with the European Convention on Animal Care. The study protocol was approved by the local animal committee.

\section{Study Design}

Eleven domestic pigs were randomly divided into 2 groups: The cisplatin (CDDP)-solution group $(\mathrm{n}=6)$ received $90 \mathrm{mg}$ of cisplatin per square meter of body surface area diluted in $500 \mathrm{~mL}$ of sodium chloride $0.9 \%$ into the chest cavity according to the dosages described in the literature. ${ }^{5}$ The CDDP-fibrin group $(\mathrm{n}=5)$ received $5 \mathrm{mg}$ of cisplatin combined to the fibrin sealant Vivostat on a predefined area of $10 \times 10 \mathrm{~cm}$ of the chest wall. Vivostat (Vivolution) is a commercially available autologous surgical fibrin sealant and CDDP fibrin was prepared as described previously. ${ }^{12}$ For comparison of both groups, a dose- and application surface-dose correction of the CDDP concentrations measured was performed (see Pharmacologic Analysis).

\section{Surgery}

Before intracavitary application of CDDP, a left-sided pneumonectomy and subtotal pleurectomy-imitating the procedure of extrapleural pneumonectomy in humans-was performed via lateral thoracotomy in the fifth intercostal space. According to the randomization, CDDP was administered as a solution or sprayed in combination with fibrin throughout a trocar in the left hemithorax. Cisplatin-fibrin was applied on the inside of the dorsolateral chest cavity. The animals were kept under narcosis for 4 hours. At time points 2 and 4 hours after CDDP administration, videoassisted thoracic surgery biopsy specimens ( 2 superficial and 2 deep) from the chest wall were obtained after removal of the CDDP-fibrin film. A chest tube was placed temporarily and was removed before extubation. The animals were under veterinarian supervision during the whole observation period of 5 days, and a well-being score measuring body temperature, breathing and heart rate, feeding and drinking behavior, mobility of the animals, posture of the animals, pain signs, and the use of analgesics (maximum of 5 points per criterion) was assessed several times a day. On days 2 and 5 after intracavitary application of cisplatin, we obtained pleural biopsy specimens by video-assisted access from the left chest wall in deep sedation. On the fifth day the animals were humanely killed in deep general anesthesia.

\section{Samples}

Tissue samples (superficial and deep) of the chest wall were obtained 2 and 4 hours and 2 and 5 days after intracavitary application of cisplatin after removal of the CDDP-fibrin film. The use of special thoracoscopic forceps guaranteed standardized biopsy specimens of 2 different depths: superficial $(\sim 0.5 \mathrm{~cm}$ containing tissue of the endothoracic fascia and superficial layer of intercostal muscle) and deep $(\sim 1.5 \mathrm{~cm}$ containing tissue of the deeper layers of intercostal muscle). In detail, in cisplatin-solution animals, we took biopsy tissue both from the lateral and from the dorsal chest, mostly from the region of the sixth to eighth intercostal space to reach parts of the chest wall that were covered with the solution. In cisplatin-fibrin animals, we first removed the CDDP-fibrin sealant film over the region selected for biopsy and subsequently performed the biopsy procedures in the same way that we did in the other group. The biopsy tissues were frozen at $-20^{\circ} \mathrm{C}$ for later duplicate platinum level determination.

Blood samples were taken before treatment (baseline), at 1, 2, 4, 8, 12, 24 hours, and 2 and 5 days after intracavitary cisplatin instillation. The blood was coagulated in serum tubes and was centrifuged. The supernatant was frozen at $-20^{\circ} \mathrm{C}$ for later duplicate platinum level determination. Laboratory findings included determination of hemoglobin, hematocrit, white blood cells, platelets, urea, and creatinine. Liver parameters (lactate dehydrogenase, aspartate aminotransferase, glutamate-pyruvate transaminase, and alkaline phosphatase) were determined before the operation, 8 and 24 hours after cisplatin instillation, and on days 2 and 5 .

Urine samples were collected before treatment and from 2- and 4-hour pools via urinary catheter. The samples were frozen at $-20^{\circ} \mathrm{C}$ for later duplicate platinum level determination.

The level of total platinum in all samples was measured by inductively coupled plasma sector field mass spectrometric detection with a matrixmatched calibration procedure at the Department of Inorganic Chemistry, ETH, as described previously. ${ }^{14,15}$

\section{Pharmacologic Analysis}

To guarantee comparability of the results between both treatment groups, we calculated a dose and surface correction of the concentrations measured in the serum and chest wall tissue as follows: the measured CDDPconcentration value was divided by a variable $\mathrm{x}$. For the CDDP-fibrinreceiving group, $x$ equaled the applied amount of CDDP $(5 \mathrm{mg})$ divided by the CDDP-applied area of the chest wall $(10 \cdot 10 \mathrm{~cm})=>\mathrm{x}=0.05$. For the CDDP-solution group, $\mathrm{x}$ equaled the applied amount of CDDP (which was calculated for each pig according to body surface area) divided by the total pleural surface area, which was measured with the help of volumetry on a computed tomographic scan of a $50-\mathrm{kg}$ pig as $900 \mathrm{~cm}^{2}$ because there were no data available in the literature about the pleural surface of pigs. Inasmuch as the anatomic geometry of the swine chest cavity is similar to a pyramid, the arithmetic formula for pyramid was used to approximate the pleural surface, but using only 3 planes. The length of the base corresponds to the distance from the spine to the lateral side of the pleura, and the height corresponds to the distance from apex to base.

All CDDP concentrations at the different time points were compared between both groups, the maximum concentration determined as well as the area under the concentration-time curve from 0 to 120 hours after surgery. For analysis of the excreted CDDP amount in the urine, the measured CDDP concentrations in urine samples were multiplied by the measured volume of urine excreted in the different time periods ( $0-2$ hours, $2-4$ hours).

\section{Statistical Analysis}

Sample size was calculated with the help of the program "nQueryAdvisor" (Elasthoff, 1995). A sample size of 5 animals in each group would have $80 \%$ power to detect a difference in means of 2.1 standard deviations using a 2-group $t$ test with a .052 -sided significance level. Statistical analysis was carried out using the software package SPSS (version 12.0; SPSS Inc, Chicago, Ill), and WinNonlin 5.2 (Pharsight, Mountain View, Calif) 
was used for pharmacokinetic calculations. Data are given as mean with standard deviation or, to account for ln-normal distribution of concentration-dependent data, as geometric mean and geometric coefficient of variation $(\mathrm{CV})$. This was calculated as $\mathrm{CV}=100 \cdot \sqrt{ }\left(\mathrm{e}^{\sigma 2}-1\right)$, where e is Euler's number and $\sigma^{2}$ is the mean square error of analysis of variance (ANOVA) testing. For statistical analysis and pharmacokinetic comparison between groups, concentration-dependent data were $\mathrm{ln}$ transformed. A repeated-measures ANOVA was carried out to test for a difference between the groups over the whole time period of measurements.

\section{RESULTS}

\section{Animal Well-Being and Analysis of the Blood}

All animals survived the experiment until the autopsy at day 5 except for 1 animal of the cisplatin-solution group, which died at the end of surgery owing to proven bacterial sepsis. This animal was replaced, but the values measured during the first 4 hours of the deceased animal were not excluded from analysis. The animals of both treatment groups were comparable. No significant differences in the preoperative weight were observed: cisplatin-fibrin group $(n=5)$, with a mean weight of $50.7 \mathrm{~kg}$ (standard deviation, 3.44), and control cisplatin-solution group $(\mathrm{n}=6)$, with a mean weight of 52.3 (standard deviation, 3.9); $P>0.05$. The animals' well-being during the experiments was evaluated on a daily basis in a repeated manner by means of a score system that showed a significant difference between both groups from the day of surgery up to the day of planned death. The animals of the cisplatin-fibrin group had a significantly better postoperative course than did the control group (repeated-measures ANOVA, $P<.001$ for interaction).

The analysis of leukocytes, hematocrit, thrombocytes, and liver enzymes (lactate dehydrogenase, aspartate aminotransferase, alkaline phosphatase, and gamma-glutamyl transferase), revealed no significant differences between the groups $(P>.05)$. The number of leukocytes increased after treatment with a maximum at 8 hours for the CDDP-fibrin group and at 12 hours for the CDDP-solution group. Thrombocyte counts remained almost unchanged up to day 5 after treatment, at which point they showed a steep increase in both groups. The hematocrit value was increased in the time period of 8 hours up to day 1 after treatment in both groups. Significant difference between both groups was observed concerning retention values, such as creatinine and urea (Figure 1): creatinine values for the CDDP-fibrin group were significantly lower from the time point 8 hours until 5 days after treatment (repeated-measures ANOVA, $P<.0001$ for interaction). The same significant difference was observed for urea values, with an important increase from 12 hours on until day 5 in the CDDP-solution-treated group (repeatedmeasures ANOVA, $P<.0001$ for interaction).

\section{CDDP Concentration in the Tissue of the Chest Wall}

The dose- and application surface-corrected geometric mean concentration of CDDP in superficial tissue of the chest wall reached maximum values 2 hours after the appli-

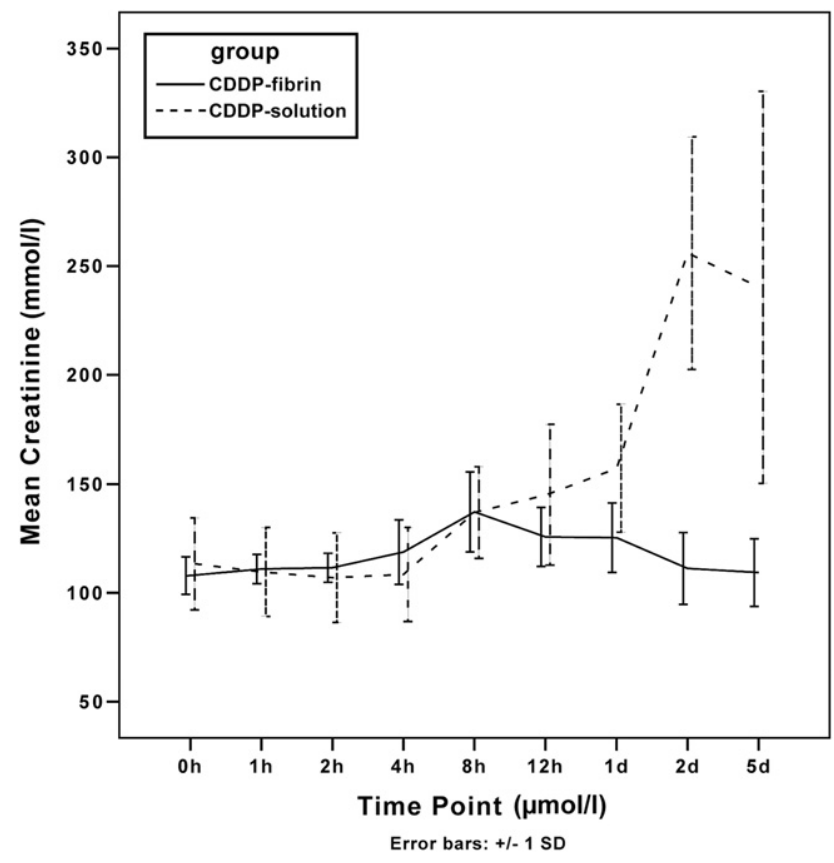

FIGURE 1. Creatinine ( $\mathrm{mmol} / \mathrm{L})$ measured in the plasma at several time points in both groups: CDDP-fibrin and CDDP-solution. Data are log transformed and given as mean and standard deviation.

cation. In animals treated with CDDP-fibrin, a mean maximum of $504 \mathrm{mg} / \mathrm{kg}$ was observed (geometric CV, 88\%), whereas animals receiving CDDP-solution had a mean maximum tissue concentration of $249 \mathrm{mg} / \mathrm{kg}$ (CV 261\%). Although the point estimate of the ratio of the values in CDDP-fibrin-treated animals over the values in CDDPsolution-treated animals showed roughly 2-fold higher concentrations obtained with CDDP-fibrin (point estimate $202 \%$ ), the $90 \%$ confidence intervals $(90 \% \mathrm{CI})$ included unity $(55 \%-751 \%)$, and therefore differences were not statistically significant $(P>.05)$. Five days after the application, mean concentrations in superficial tissues of the chest wall were $73 \mathrm{mg} / \mathrm{kg}$ (CV 216\%) and $22 \mathrm{mg} / \mathrm{kg}$ (CV $427 \%$ ) in CDDP-fibrin- and CDDP-solution-treated animals, respectively (point estimate 332\%, 90\% CI $55 \%-2015 \%$ ). CDDP in superficial chest wall tissue tended to be higher and longer, respectively, in the group of animals treated with CDDP-fibrin $(P>.05)$ (Table 1$)$.

TABLE 1. Geometric means and coefficients of variation (CV) of maximum measured CDDP concentration (Cmax), and area under the curve (AUC), measured in the superficial tissue of the chest wall of both groups CDDP-fibrin and CDDP-solution $(P>.05)$

\begin{tabular}{lcc}
\hline $\begin{array}{c}\text { Geometric } \\
\text { mean }(\mathbf{C V} \%)\end{array}$ & $\begin{array}{c}\text { Cmax 2 hours } \\
(\mathbf{m g} / \mathbf{k g})\end{array}$ & $\begin{array}{c}\text { AUC } \\
(\mathbf{m g} / \mathbf{k g} \cdot \mathbf{h})\end{array}$ \\
\hline CDDP-fibrin & $504(88)$ & $27,962(54)$ \\
CDDP-solution & $249(261)$ & $9,784(691)$ \\
\hline
\end{tabular}


The differences in dose- and application surfacecorrected area under the curve of CDDP in deep tissue of the chest wall between the groups were not so important as observed in the superficial tissues $(P>.05)$ (Table 2). This was also reflected in comparably high maximum CDDP concentrations: $72 \mathrm{mg} / \mathrm{kg}(1269 \%)$ for CDDPfibrin after 2 hours and $73 \mathrm{mg} / \mathrm{kg}(1303 \%)$ for CDDPsolution after 4 hours. Nevertheless, the point estimate for the ratio of CDDP concentrations in deep tissues 5 days after the application of $134 \%(90 \%$ CI $31 \%-574 \%)$ showed that CDDP concentrations in deep tissue tended to be higher in the cisplatin-fibrin group.

\section{CDDP Serum Concentrations}

In serum, the dose- and application surface-corrected exposure toward CDDP (area under the concentration-time curve from 0-120 hours after surgery) was clearly and significantly lower in animals treated with CDDP-fibrin in comparison with animals treated with CDDP-solution: $69 \mathrm{mg} / \mathrm{L} \cdot \mathrm{h}(\mathrm{CV} 28 \%)$ versus $756 \mathrm{mg} / \mathrm{L} \cdot \mathrm{h}(\mathrm{CV}$ $110 \%$ ), point estimate of the ratio of means in the fibrintreated group over the means in the solution-treated group: $9 \%(90 \%$ CI, $4 \%-20 \%)(P<.0005)$ (Figure 2). The same was true for the maximum values of CDDP in serum, with a point estimate of $15 \%(90 \% \mathrm{CI}, 10 \%-25 \%)$, meaning a reduction by $85 \%$ of maximum CDDP concentrations in the serum for the animals treated with cisplatin-fibrin.

\section{CDDP Amount Excreted in the Urine}

The amount of CDDP excreted in urine during 0 to 2 hours and 2 to 4 hours after CDDP application was significantly lower in the CDDP-fibrin-treated animals than in the cisplatin-solution-treated pigs $(P<.0001)$ (Figure 3$)$.

\section{DISCUSSION}

In this preclinical animal study, CDDP chest wall tissue concentration after CDDP-fibrin treatment was increased whereas systemic CDDP concentration was significantly reduced. The reduced systemic drug concentrations are reflected in significantly lower serum creatinine and urea levels as well as better well-being scores in animals treated with CDDP-fibrin. Together with the results of our previous small animal studies in our mesothelioma recurrence model, where intracavitary cisplatin-fibrin and cisplatinsolution are equally efficient in reducing tumor recurrence,

TABLE 2. Geometric means and coefficients of variation (CV) of maximum CDDP concentration (Cmax) and the area under the curve (AUC) in the deep tissue of the chest wall of both groups (CDDP-fibrin and CDDP-solution, $P>$.05)

\begin{tabular}{lcc}
\hline $\begin{array}{c}\text { Geometric } \\
\text { mean }(\mathbf{C V} \%)\end{array}$ & $\begin{array}{c}\text { Cmax 2 hours } \\
(\mathbf{m g} / \mathbf{k g})\end{array}$ & $\begin{array}{c}\text { AUC } \\
(\mathbf{m g} / \mathbf{k g} \cdot \mathbf{h})\end{array}$ \\
\hline CDDP-fibrin & $72(1269)$ & $4408(101)$ \\
CDDP-solution & $52(540)$ & $3875(254)$ \\
\hline
\end{tabular}

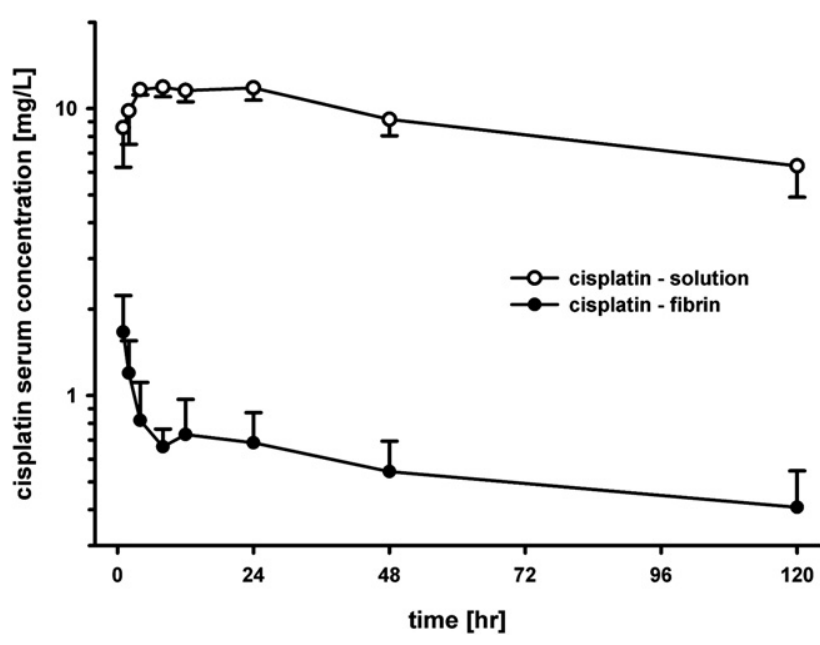

FIGURE 2. Geometric mean CDDP concentrations ( $\mathrm{mg} / \mathrm{L}$ ) in serum measured at several time points in both groups of animals treated with CDDPfibrin and CDDP-solution, respectively. Data are given as geometric mean and geometric standard deviations. Please note the logarithmic scale of the y-axis.

the finding of the present study demonstrates a clear advantage of CDDP-fibrin inasmuch as rate and severity of systemic adverse events can be reduced while local cytotoxic concentrations are at least maintained.

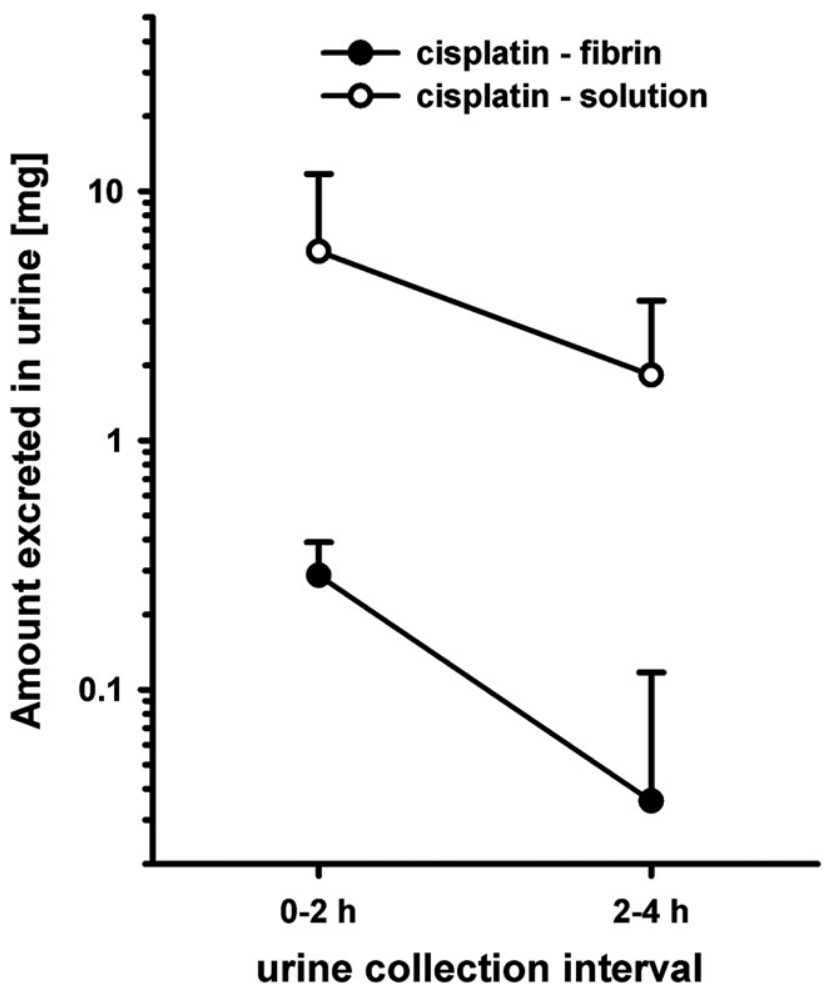

FIGURE 3. Amount of CDDP excreted in urine measured during 2 time periods from 0 to 2 and from 2 to 4 hours after CDDP application. Data are given as geometric means and geometric standard deviations. Please note the logarithmic scale on the $y$-axis. 
Intracavitary treatment with CDDP-fibrin in contrast to CDDP-solution, as used by many other groups, ${ }^{3-5,7-10}$ might be a therapy option to decrease treatment-related morbidity of the latter. Mortality rates up to $11 \%$ and a risk of up to $57 \%$ for renal toxicity and $13 \%$ for deep venous thrombosis and pulmonary embolus ${ }^{3,4,10}$ can be explained by rapid absorption of cisplatin from the pleural cavity with high systemic drug levels when applied as a solution. Several renal protective measures have been undertaken for this problem, for example, postoperative hydration, which cannot be performed in the setting of extrapleural pneumonectomy to avoid excessive contralateral fluid retention. Other measures investigated are the application of amifostine, ${ }^{3}$ with still inadequate renal protection; also, after the combination of amifostine with sodium thiosulfate, the overall risk of renal dysfunction is $14 \% .{ }^{10}$ The use of cisplatin bound to a fibrin carrier might prevent the problem of renal toxicity by preventing rapid absorption. The fibrin sealant acts as a depot for cisplatin, from which it is released during a longer time period than after application of a cisplatin solution into the pleural cavity, which is rapidly absorbed. If we assume that the transport to deeper tissues (and then to the blood) is mainly a first-order diffusion process and not saturated by the concentrations achieved, but that the application is maintained for a longer time with CDDP-fibrin, a higher local exposure (area under the curve) after the application of the fibrin-cisplatin mixture would result from the slower absorption of CDDP from the fibrin sealant. Additionally, local concentrations are higher, because cisplatin readily binds to proteins and is presumably thereby better retained at the application site if concentrations are maintained for a longer time. Inasmuch as we corrected for both dose and application surface, the depot effect and the more effective tissue protein binding when cisplatin exposure is maintained for a longer time period could explain higher local concentrations and lower systemic exposure, since the liberation of cisplatin from protein binding is a very slow process. This is underlined by the fact that we reach with cisplatin-fibrin at least the same local tissue concentrations as with cisplatin-solution, although the applied amount of cisplatin per square centimeter is lower. One might think about even further increasing the cisplatinapplication dosage with the help of cisplatin-fibrin. Cisplatin shows cytotoxic growth arrest in vitro on human mesothelioma cell lines in the micromolar range. The concentrations obtained in the superficial and deep chest wall tissue in both groups exceed the concentration necessary to produce necrosis $(1 \mu \mathrm{mol} / \mathrm{L}=300 \mu \mathrm{g} / \mathrm{L})$ by more than factor 1000. The cytotoxic potential of CDDP-fibrin was also tested in vitro after cisplatin elution in solution (data not shown) on a human mesothelioma cell line beforehand, and we observed a dose-dependent cell killing. Thus, eventually this compound can be used to apply even higher dosages than the one tested in clinical studies ranging up to $225 \mathrm{mg} / \mathrm{m}^{2}$ as maximum tolerated dose. ${ }^{4}$ With these concentrations, Sugarbaker and associates have proven distinct survival advantage of 26 months for those patients receiving high-dose cisplatin lavage. ${ }^{4}$ Another difference of our protocol in contrast to other groups is that we decided not to add hyperthermia to our treatment protocol. Our reason was that hyperthermia influences the process of platelet activation, ${ }^{16}$ fibrin formation, ${ }^{17}$ and fibrinolysis ${ }^{18}$ - complications for which patients with malignant pleural mesothelioma are already at high risk owing to the malignancy itself. Although The Brigham and Women's Hospital group looks back now on a great experience with hyperthermic intraoperative chemotherapy and refined the protocol more and more over the past years, the procedure itself remains complex, with a 60minute lavage, and is not easy for other centers to copy. Therefore, our application system with a spray-pen that covers the whole chest cavity or distinct areas with cisplatin film seems to facilitate the practical aspect.

A critical point of the study is the variability of the data reflected in large coefficients of variation. This variation, especially in the biopsy tissues, can be explained by the modus of tissue concentration assessment. Although we always used the same forceps and followed a standardized scheme of biopsy, there might be a certain interexamination variability. The variability of the data is probably also due to the limited sample size of only 5 animals per group. Nevertheless, there is a clear trend recognizable of not only significantly decreased serum but also increased CDDP tissue concentration after CDDP-fibrin treatment. Another point for discussion is that the fibrin sealant applies the chemotherapy to a specific area covered by the sealant, whereas the solution reaches the entire pleural cavity. As already mentioned, the dose- and application-surface normalization was intended to make the 2 treatment strategies comparable in terms of local tissue concentrations and systemic exposure irrespective of the dose and the absorption surface.

If one considers our results of previous small animal studies and the clinical studies of other groups, the use of intrapleural chemotherapy is an attractive approach to improve local tumor control because $50 \%$ reduction of ipsilateral recurrence was proven (Tilleman and associates ${ }^{10}: 34 \%$ ) in comparison with historical controls (Baldini and associates $^{19}: 67 \%$ ). The CDDP-fibrin approach seems to be an attractive alternative to decrease renal toxicity and may be a vehicle to reach increased local toxicity. On the one hand, CDDP-fibrin can be used clinically as an alternative treatment option combined with less radical procedures such as pleurectomy/decortication for patients who are unlikely to benefit from an aggressive multimodal regimen (eg, patients with insufficient cardiopulmonary reserve, advanced age, or disease pattern). Alternatively, CDDP-fibrin can be used as an additional treatment option if, during radical surgery, zones potentially at risk for recurrence are identified and "prophylactically" treated with topical 
cisplatin-fibrin, which has to be further evaluated. In a next step, we will further evaluate the application of cisplatinfibrin on the whole surface of the chest cavity before starting a clinical phase I study.

We thank the veterinarians, Dr Kronen and Dr Sidler, as well as Boris Leskosek and Flora Nicholls for assistance and Daniel Demierre for technical equipment during the animal experiments. We thank Dr Latkoczy and Professor Günther for determination of cisplatin in tissue and serum. We thank PD Dr Felley-Bosco for in vitro studies of cisplatin fibrin and critical reading of the manuscript. We thank Dr Thomas Frauenfelder for the volumetry of pigs' pleural surface.

\section{References}

1. Krug LM, Pass HI, Rusch VW, Kindler HL, Sugarbaker DJ, Rosenzweig KE, et al. Multicenter phase II trial of neoadjuvant pemetrexed plus cisplatin followed by extrapleural pneumonectomy and radiation for malignant pleural mesothelioma. J Clin Oncol. 2009;27:3007-13.

2. Weder W, Stahel RA, Bernhard J, Bodis S, Vogt P, Ballabeni P, et al. Multicenter trial of neo-adjuvant chemotherapy followed by extrapleural pneumonectomy in malignant pleural mesothelioma. Ann Oncol. 2007;18:1196-202.

3. Zellos L, Richards WG, Capalbo L, Jaklitsch MT, Chirieac LR, Johnson BE, et al. A phase I study of extrapleural pneumonectomy and intracavitary intraoperative hyperthermic cisplatin with amifostine cytoprotection for malignant pleural mesothelioma. J Thorac Cardiovasc Surg. 2009;137:453-8.

4. Richards WG, Zellos L, Bueno R, Jaklitsch MT, Janne PA, Chirieac LR, et al. Phase I to II study of pleurectomy/decortication and intraoperative intracavitary hyperthermic cisplatin lavage for mesothelioma. J Clin Oncol. 2006;24: 1561-7.

5. Rusch V, Saltz L, Venkatraman E, Ginsberg R, McCormack P, Burt M, et al. A phase II trial of pleurectomy/decortication followed by intrapleural and systemic chemotherapy for malignant pleural mesothelioma. J Clin Oncol. 1994;12: 1156-63.

6. Chang MY, Sugarbaker DJ. Innovative therapies: intraoperative intracavitary chemotherapy. Thorac Surg Clin. 2004;14:549-56.

7. Lee JD, Perez S, Wang HJ, Figlin RA, Holmes EC. Intrapleural chemotherapy for patients with incompletely resected malignant mesothelioma: the UCLA experience. J Surg Oncol. 1995;60:262-7.

8. Colleoni M, Sartori F, Calabro F, Nelli P, Vicario G, Sgarbossa G, et al. Surgery followed by intracavitary plus systemic chemotherapy in malignant pleural mesothelioma. Tumori. 1996;82:53-6.

9. Rice TW, Adelstein DJ, Kirby TJ, Saltarelli MG, Murthy SR, Van Kirk MA, et al. Aggressive multimodality therapy for malignant pleural mesothelioma. Ann Thorac Surg. 1994;58:24-9.

10. Tilleman TR, Richards WG, Zellos L, Johnson BE, Jaklitsch MT, Mueller J, et al. Extrapleural pneumonectomy followed by intracavitary intraoperative hyperthermic cisplatin with pharmacologic cytoprotection for treatment of malignant pleural mesothelioma: a phase II prospective study. J Thorac Cardiovasc Surg. 2009;138:405-11.

11. Rusch VW, Niedzwiecki D, Tao Y, Menendez-Botet C, Dnistrian A, Kelsen D, et al. Intrapleural cisplatin and mitomycin for malignant mesothelioma following pleurectomy: pharmacokinetic studies. J Clin Oncol. 1992;10:1001-6.

12. Lardinois D, Jung FJ, Opitz I, Rentsch K, Latkoczy C, Vuong V, et al. Intrapleural topical application of cisplatin with the surgical carrier Vivostat increases the local drug concentration in an immune-competent rat model with malignant pleuromesothelioma. J Thorac Cardiovasc Surg. 2006;131:697-703.

13. Opitz I, Lardinois D, Arni S, Hillinger S, Vogt P, Odermatt B, et al. Local recurrence model of malignant pleural mesothelioma for investigation of intrapleural treatment. Eur J Cardiothorac Surg. 2007;31:773-8.

14. Bettinelli M. ICP-MS determination of Pt in biological fluids of patients treated with antitumor agents: evaluation of analytical uncertainty. Microchem J. 2005; 79:357-65.

15. Zimmermann S, Menzel CM, Berner Z, Eckhardt J-D, Stüben D, Alt F, et al. Trace analysis of platinum in biological samples: a comparison between sector field ICP-MS and adsorptive cathodic stripping voltammetry following different digestion procedures. Anal Chim Acta. 2001;439:203-9.
16. Take H, Kubota K, Tamura K, Kurabayashi H, Shirakura T, Miyawaki S, et al. Activation of circulating platelets by hyperthermal stress. Eur J Med Res. 1996; $1: 562-4$

17. Pivalizza EG, Koch SM, Mehlhorn U, Berry JM, Bull JMC. The effects of intentional hyperthermia on the Thrombelastograph and the Sonoclot analyser. Int $J$ Hyperthermia. 1999;15:217-23.

18. Tamura K, Kubota K, Kurabayashi H, Shirakura T. Effects of hyperthermal stress on the fibrinolytic system. Int J Hyperthermia. 1996;12:31-6.

19. Baldini EH, Recht A, Strauss GM, DeCamp MM Jr, Swanson SJ, Liptay MJ, et al. Patterns of failure after trimodality therapy for malignant pleural mesothelioma. Ann Thorac Surg. 1997;63:334-8.

\section{Discussion}

Dr W. Roy Smythe (Temple, Tex). I appreciate the opportunity to comment on this paper and I also thank the authors for sending the manuscript ahead of time. Walt Whitman said the art of art, the glory of expression, and the sunshine of the light of letters is simplicity. The use of intrapleural chemotherapy for an intrapleural malignancy suggests Whitman's simplicity; however, this technique, which was originally experimentally pioneered by Valerie Rusch and colleagues at Memorial Sloan-Kettering and David Sugarbaker and his colleagues at Brigham, followed by more than a decade of clinical use, pioneering clinical use by the Brigham group, has been less than anticipated. In all likelihood, most of the issues with this approach have related to the lack of persistence of the drug at the site and then the associated toxicities, which your group has mentioned. I think this study, on the other hand, is very simple and elegant and begins to address some of these issues. I have the following questions: First, is there any evidence that the fibrin carrier interacts with the drug, either augmenting or attenuating the effect of the drug? I noticed that the snake-derived venom, batroxobin, is used in this formulation. Batroxobin, for example, is a serine protease, which has been shown itself in many animal models of malignancy to have an antitumor effect.

Dr Opitz. We could not address this in the pig model because this is not a tumor model. However, in our previous small animal studies, we also analyzed a group treated with fibrin alone and we observed no antitumor efficacy with fibrin alone.

Dr Smythe. Very good. The second question is, if the major mechanism of action here is the film holding the drug in place for a period of time, that would suggest that other drugs or even biological agents might be applicable to this model. Have you tried those in the past in any of your models?

Dr Opitz. We have tried chemokines that were topically applied and we have tried CpG-ODNs, but the efficacy was not higher with these drugs if combined to fibrin in comparison to cisplatin, as it is already very active. However, there are a lot of options to test this in the future. Any drug can be combined with fibrin.

Dr Smythe. The last question is, one of the other issues with intrapleural chemotherapy, one of the reasons some groups have used heated chemotherapy rather than just simple installation, is depth of penetration of the drug. A group that I previously worked with showed in 2005 that if you use a liposomal formulation of cisplatin, you can get better depth of penetration in tumor. Have you considered perhaps using this fibrin carrier in combination with a lipid carrier to maybe titrate depth of penetration?

Dr Opitz. We discussed this issue during the setup of our protocol. However, in the literature, there is really a high rate of 
pulmonary embolism and deep venous thrombosis in the clinical phase I and phase II studies, and it has been demonstrated that hyperthermia leads to fibrin formation or disorganization of the whole coagulation cascade. We decided for our protocol not to use hyperthermia.

Dr Steven J. Mentzer (Boston, Mass). A poorly recognized biological characteristic of cisplatin is that it can generate an immunologic response by directly modifying class I peptides. It is possible that some of the clinical response you observe may be immunologically mediated? The potential immunologic activity of cisplatin raises concerns about the biological availability of cisplatin after fibrin conjugation. Do you have any data that cisplatin is biologically active?

Dr Opitz. No, we do not have any data.

Access to The Journal of Thoracic and Cardiovascular Surgery Online is reserved for print subscribers!

Full-text access to The Journal of Thoracic and Cardiovascular Surgery Online is available for all print subscribers. To activate your individual online subscription, please visit The Journal of Thoracic and Cardiovascular Surgery Online, point your browser to http://www.mosby.com/itcvs, follow the prompts to activate your online access, and follow the instructions. To activate your account, you will need your subscriber account number, which you can find on your mailing label (note: the number of digits in your subscriber account number varies from 6 to 10 ). See the example below in which the subscriber account number has been circled:

\section{Sample mailing label}

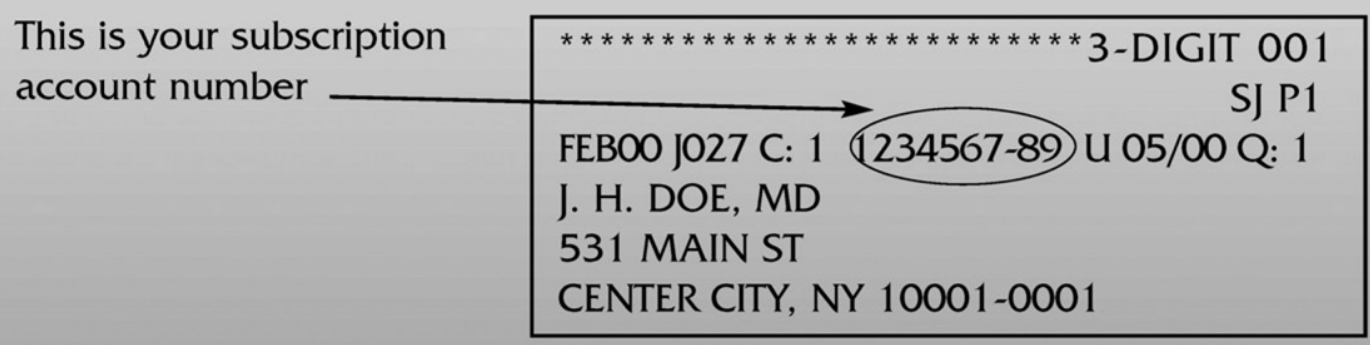

Personal subscriptions to The Journal of Thoracic and Cardiovascular Surgery Online are for individual use only and may not be transferred. Use of The Journal of Thoracic and Cardiovascular Surgery Online is subject to agreement to the terms and conditions as indicated online. 